\title{
Phénoménologie et anthropologie
}

\section{Françoise Dastur}

\section{OpenEdition}

\section{Journals}

Édition électronique

URL : http://journals.openedition.org/alter/369

DOI : 10.4000/alter.369

ISSN : 2558-7927

\section{Éditeur :}

Association ALTER, Archives Husserl (CNRS-UMR 8547)

\section{Édition imprimée}

Date de publication : 1 novembre 2015

Pagination : 28-45

ISBN : 978-2-9550449-7-9

ISSN : 1249-8947

\section{Référence électronique}

Françoise Dastur, «Phénoménologie et anthropologie », Alter [En ligne], 23 | 2015, mis en ligne le 01 décembre 2017, consulté le 20 avril 2019. URL : http://journals.openedition.org/alter/369 ; DOI : 10.4000/alter.369 


\section{PHÉNOMÉNOLOGIE ET ANTHROPOLOGIE ${ }^{1}$}

Françoise Dastur

Phénoménologie et anthropologie : ces deux termes sont-ils antinomiques ou peut-on, sans cesser de se réclamer de Husserl, voire de Heidegger, les deux figures tutélaires du «mouvement » phénoménologique, déployer aujourd'hui le projet d'une anthropologie phénoménologique ? Telle est la question ambitieuse à laquelle on se propose ici de tenter de répondre de manière au moins programmatique. Il est pour cela nécessaire de commencer par dessiner à grands traits le contexte dans lequel elle se pose. Que signifie en effet le retour, depuis une bonne vingtaine d'années, de la question anthropologique, alors même que cette question s'était vue bannie pendant toute la période de ce que l'on a pu nommer la "pensée 68 ", caractérisée comme « anti-humaniste $»^{2}$ ?

À cet égard en effet, ce n'est pas seulement ce représentant de ce que les auteurs de La pensée 68 nomment le «nietzschéisme français » qu'est Michel Foucault qu'il faut invoquer, mais aussi Jacques Derrida, autour du travail duquel un grand colloque fut organisé en 1980 sur le thème des «Fins de l'homme $»^{3}$. Certes c'est bien dans Les mots et les choses, publié en 1966, qu'est annoncée, après celle de Dieu, la mort de l'homme lui-même, par celui qui fut pourtant, quelques années auparavant, fortement marqué par «l'anthropologie phénoménologique » de Binswanger appellation à laquelle ce dernier se rallia avant de lui substituer en 1942 celle de Daseinsanalyse. Rappe-

\footnotetext{
${ }^{1}$ Une première version anglaise de ce texte a paru sous le titre « Phenomenology and Anthropology ", in Philosophy today, vol. 54, C. Willett et L. Lawlor (éd.), 2010, p. 5-14.

${ }^{2}$ L. Ferry et A. Renault, La pensée 68, Essai sur l'anti-humanisme contemporain, Paris, Gallimard, 1988.

${ }^{3}$ Les fins de l'homme, À partir du travail de Jacques Derrida, colloque de Cerisy, 23 juillet-2 août 1980, Paris, Galilée, 1981.
} 
lons que c'est Michel Foucault qui préfaça en 1954 la traduction française de l'essai de Binswanger, "Le rêve et l'existence», qui porte précisément en exergue une phrase de Kierkegaard qui dit: «Il convient plutôt de s'attacher à ce que signifie : être un homme ».

Michel Foucault ne fut effectivement pas le seul à affirmer, en 1966, que « de nos jours on ne peut plus penser que dans le vide de $l^{\prime}$ homme disparu $»^{4}$. Car si la critique foucaldienne de l'humanisme s'appuie sur celle de la métaphysique de la subjectivité telle qu'elle a été élaborée par Nietzsche, mais aussi par Heidegger, dont Foucault reprend, sans le dire explicitement, «l'analytique de la finitude $»^{5}$, et si elle est visiblement dirigée contre l'existentialisme de Sartre et de Merleau-Ponty, celle que reprend à son compte Derrida, dans sa conférence new-yorkaise de 1968 intitulée "Les fins de l'homme " vise plus directement Husserl et Heidegger. Derrida y décrit en effet le contexte historique dans lequel il se situe alors comme l'époque du reflux anti-humaniste et anti-anthropologiste venant après la vague humaniste et anthropologiste qui avait recouvert la philosophie française pendant la période existentialiste ${ }^{6}$. Il y souligne que la critique de l'anthropologisme que l'on trouve chez Hegel, Husserl et Heidegger continue de demeurer complètement inaperçue, mais il reconnaît cependant qu'une "justification profonde » et une «nécessité souterraine » conduisent les intellectuels d'aujourd'hui à "amalgamer » non seulement Husserl, mais aussi Hegel et Heidegger, à la «vieille métaphysique humaniste » et il entreprend de montrer qu'il est possible de trouver une "relève» de l'homme chez ces trois penseurs". Derrida signe ici, de manière encore plus nette que dans ses textes précédents, sa rupture avec la pensée du phainesthai, liée chez Hegel et Husserl, à celle d'une téléologie de la raison, de sorte que chez ce dernier en particulier, "la critique de l'anthropologisme empirique n'est que l'affirmation d'un humanisme transcendantal $»^{8}$. Mais il n'en prend pas moins également ses distances par rapport à la phénoménologie herméneutique de Heidegger, où la question de l'apparaître est inséparable à ses yeux d'une " pensée de la présence » qui se déploie comme pensée du propre de l'homme 9 .

Pourtant, contrairement à ce qu'annonçaient conjointement à la fin des années soixante Foucault et Derrida, à savoir la « fin prochaine »

\footnotetext{
${ }^{4}$ M. Foucault, Les mots et les choses, Paris, Gallimard, 1966, p. 353.

${ }^{5}$ Ibid., p. 323 sq.

${ }^{6}$ Cf. J. Derrida, Marges de la philosophie, Paris, Minuit, 1972, p. 140-141.

${ }^{7}$ Ibid., p. 142.

${ }^{8}$ Ibid., p. 147.

${ }^{9}$ Ibid., p. 159-160.
} 
de cette «invention de date récente » qu'est $\mathrm{l}^{\prime}$ homme ${ }^{10}$, à laquelle aurait dû s'ajouter aussi la disparition de la phénoménologie, cette dernière est cependant parvenue à ressusciter de ses cendres, un peu comme ce Phénix, symbole de l'esprit, qu'invoquait Husserl à la fin de sa conférence de Vienne de mai 1935 qui traitait justement de «La crise de l'humanité européenne ». Preuve en est la multiplication des écrits consacrés à la pensée husserlienne par de jeunes chercheurs au cours des dernières décennies, recherches certes non exclusivement, mais très souvent consacrées à cette dernière philosophie de Husserl qu'on peut, nonobstant sa critique demeurée constante de l'anthropologisme, qualifier néanmoins $\mathrm{d}^{\prime}$ « humaniste ». C'est donc de cette dernière philosophie de Husserl qu'il faut repartir pour tenter une première approche des liens qui pourraient relier anthropologie et phénoménologie.

Certes l'association du terme d' « humanisme » au nom de Husserl peut au premier abord sembler impropre, puisque la phénoménologie en tant que nouvelle forme de philosophie a trouvé son point de départ dans la critique impitoyable du psychologisme, du relativisme, et de l'anthropologisme en général que Husserl développe dans le premier tome des Recherches logiques. Il y affirme en effet que les vérités logiques sont complètement indépendantes de l'esprit humain et peuvent exister sans lui, parce qu'elles ne sont pas les produits terminaux d'une genèse historique ou psychologique. L'introduction du point de vue transcendantal dans les Idées directrices pour une phénoménologie et la conversion «idéaliste » qu'il implique par rapport à la neutralité philosophique des Recherches logiques, ne signifie pourtant pas que l'homme se voit réintroduit en tant que centre des structures transcendantales. Car Husserl ne rejette pas seulement toutes les sortes d'anthropologismes empiriques, comme l'affirme Derrida, mais aussi ce qu'il nommera plus tard, dans la postface qu'il rédige en 1930 pour l'édition anglaise de ses Idées directrices « anthropologisme transcendantal» ou encore «psychologisme transcendantal » ${ }^{11}$. Ce qu'il désigne alors, sous ces vocables, c'est l'analytique existentiale heideggérienne dans laquelle il ne voit, comme l'attestent

\footnotetext{
${ }^{10} C^{\prime}$ est le passage des Mots et les choses dans lequel Foucault utilise cette expression que Derrida met en exergue de sa conférence de 1968. Cf. J. Derrida, Marges de la philosophie, op. cit., p. 131.

${ }^{11}$ E. Husserl, "Postface à mes Idées directrices pour une phénoménologie pure », trad. A. L. Kelkel, La phénoménologie et les fondements des sciences, Paris, PUF, 1993, p. 181.
} 
les annotations marginales, datant de la même époque (1930), de son exemplaire d'Être et temps, qu'une « anthropologie philosophique $»^{12}$. C'est ce qui l'avait conduit, comme il l'écrit en décembre 1929 à Roman Ingarden, à considérer qu'Être et temps, dont il achevait alors la lecture, ne peut plus être intégré à sa phénoménologie pas plus du point de vue de la méthode que du point de vue du contenu ${ }^{13}$. C'est également ce qu'il redit dans la conférence qu'il donne en juin 1931 sous le titre "Phénoménologie et anthropologie » où il dénonce, de manière plus explicite, l'anthropologisme de ceux qui, à l'intérieur du mouvement phénoménologique, ont subi l'influence de Dilthey et voudraient faire reposer le fondement de la philosophie exclusivement sur « l'homme et dans une doctrine de l'essence de son Dasein mondano-concret ${ }^{14}$. Car selon Husserl, l'homme et l'ego humain sont des réalités mondaines, c'est-à-dire des êtres relatifs, et l'être absolu, la conscience transcendantale, est une conscience non personnelle sans relation à l'homme ou à un autre être réel, comme il le soulignait en 1913 dans ses Idées directrices où il affirmait : "Il est certain qu'on peut penser une conscience sans corps, et aussi paradoxal que cela paraisse, sans âme, une conscience non personnelle, c'est-à-dire un flux vécu où ne se constitueraient pas les unités intentionnelles empiriques qui se nomment corps, âme, sujet personnel empirique, et où tous ces concepts empiriques, y compris par conséquent celui du vécu au sens psychologique (en tant que vécu d'une personne, d'un moi animé) perdraient tout point d'appui et en tout cas toute validité ${ }^{15}$.

Une telle subjectivité transcendantale peut-elle cependant être quelque chose d'autre qu'une pure abstraction ? C'est là le sens d'une remarque faite par Heidegger à Husserl en 1929 durant sa collaboration à la rédaction de l'article «Phenomenology » pour l'Encyclopædia Britannica ${ }^{16}$. Husserl y répond en affirmant que le moi transcendantal et le moi naturel ne sont pas des moi différents, ni des moi liés l'un à l'autre, mais qu'ils sont le même moi, le moi empirique se changeant en moi transcendantal par une simple conversion d'atti-

\footnotetext{
${ }^{12}$ Cf. D. Souche-Dagues, «La lecture husserlienne de Sein und Zeit » in E. Husserl, Notes sur Heidegger, D. Franck (éd.), Paris, Minuit, 1993, p. 23 et 137 sq.

${ }^{13}$ E. Husserl, Briefe an Roman Ingarden, La Haye, Martinus Nijhoff, 1968, p. 56.

${ }^{14}$ Cf. E. Husserl, «Phänomenologie und Anthropologie », Aufsätze und Vorträge (1922-1937), Husserliana XXVII, Th. Nenon et H. R. Sepp (éd.), Dordrecht/Boston/Londres, Kluwer, 1989, p. 181 ; trad. D. Franck in E. Husserl, Notes sur Heidegger, op. cit., p. 57.

${ }^{15}$ E. Husserl, Idées directrices pour une phénoménologie, trad. P. Ricœur, Paris, PUF, 1950, § 54, p. 182.

${ }^{16}$ Voir la lettre du 22 octobre 1927 de Heidegger à Husserl reproduite in E. Husserl, Notes sur Heidegger, op. cit., p. 115 sq.
} 
tude. La tâche de la phénoménologie transcendantale consiste par conséquent à révéler l'activité constitutive du moi transcendantal, laquelle demeure dissimulée à l'ego psychologique qui s'adonne directement aux objets donnés. Il n'en demeure pas moins que le rapport de l'ego transcendantal à l'ego mondain continue de faire problème, comme l'atteste la manière dont Husserl résout le problème de la constitution de l'être humain comme personne et donc comme moi empirique dans le second livre des Idées directrices. Car il continue d'y penser l'être humain, en accord avec toute la tradition métaphysique, comme l'union du sensible et de l'intelligible. C'est en effet sur la base de la nature matérielle et animale, dont traitent les deux premières sections des Ideen II, que la constitution du monde spirituel, qui fait l'objet de la troisième section, est rendue possible. L'être humain a selon lui d'abord part au règne animal et secondairement au monde spirituel. C'est donc à la nature qu'appartient par essence l'être humain, et en dépit des pages que Husserl consacre dans ce livre à l'analyse des objets spirituels et des productions culturelles, il n'est pas possible de découvrir ici les prémisses d'une véritable problématique anthropologique.

La situation est entièrement différente dans les dernières œuvres de Husserl, dans la Krisis et dans les manuscrits du milieu des années trente, où un nouveau concept de l'homme fait son émergence, en connexion avec une nouvelle définition de l'humanité. La réhabilitation de l'idée d'homme concerne donc l'homme en tant qu'être culturel, c'est-à-dire en tant qu'être de tradition et d'histoire. Husserl demeure fidèle à la définition métaphysique de l'homme comme être rationnel, comme Vernunftwesen, mais maintenant la raison est intimement reliée à l'historicité. L'homme n'est plus alors le nom d'un ego psychologique et mondain, mais, comme Derrida avait raison de le souligner, le substrat d'une raison téléologique et le corrélat d'une tâche infinie ${ }^{17}$. Car, dans cette dernière philosophie de Husserl, la théologie, la téléologie et l'anthropologie vont s'unir pour lui en une seule et même thématique. L'homme dont il s'agit alors, c'est l'homo philosophicus, parce que l'idée de la philosophie, c'est-à-dire le projet d'une science universelle, est pour Husserl le telos de l'histoire. Pour lui l'histoire est en un sens toujours histoire de la philosophie, comme c'est déjà le cas pour Hegel, mais sans fin de l'histoire et sans parousie de l'absolu dans un savoir absolu. C'est ce qui explique que son approche de l'histoire soit en réalité plus kantienne qu'hégélienne, en dépit de quelques similarités de vocabulaire, que l'on

${ }^{17}$ J. Derrida, Marges de la philosophie, op. cit., p. 146-147. 
trouve par exemple dans l'apologie que fait Husserl de l'autosuffisance de l'esprit à la fin de sa conférence de Vienne de $1935^{18}$. L'idée qui gouverne l'histoire, l'idée d'une connaissance adéquate du monde, est une « idée au sens kantien », c'est-à-dire un idéal régulateur et l'index d'une tâche infinie. Mais pour Husserl cet idéal régulateur n'est pas valable seulement, comme l'affirme Kant, pour l'intuitus derivativus de cet être fini qu'est l'homme par contraste avec l'intuitus originarius de Dieu qui possède déjà la connaissance adéquate des choses en soi.

Husserl, en rejetant l'idée d'un infini actuel et positif confère en effet au temps une nouvelle importance ontologique : l'éternité divine n'est plus pour lui la toile de fond sur laquelle s'enlève la finitude humaine, laquelle doit être mise au contraire en corrélation avec l'horizon indéfini d'un temps sans limite. C'est la raison pour laquelle les vérités logiques ne sont pas éternelles, mais bien "omnitemporelles », non pas hors du temps, mais valables pour tous les temps, car l'omnitemporalité est un mode de la temporalité et non son contraire. Toutes les idéalités, aussi bien celles qui sont liées à une réalité mondaine, comme les œuvres d'art, que celles qui sont libres de tout rapport à la subjectivité empirique, comme les entités mathématiques et logiques, sont par conséquent mondaines, du seul fait de leur surgissement historique, comme Husserl le laisse clairement entendre dans Expérience et jugement ${ }^{19}$, non au sens du relativisme qui invalide l'idée même de vérité, mais au sens d'une paradoxale historicité de la vérité elle-même. Dieu en tant que ce dieu logifié, ce porteur du logos absolu qu'il devient au sein de la tradition philosophique $^{20}, \mathrm{n}^{\prime}$ « existe » pas, ce qui veut dire qu'il n'est pas un infini actuel, mais, en tant qu'idée liée à un temps infini, il est l'horizon de l'histoire, son origine et sa finalité. Dieu n'est rien d'autre que la raison absolue venant à soi-même dans un processus infini et l'histoire elle-même peut être considérée comme « le processus de l'autoréalisation de la divinité » (Selbstrealisierungsprozess der Gottheit), comme Husserl le dit dans un manuscrit des années trente ${ }^{21}$. En fin de compte, l'idée de Dieu est pour Husserl identique à celle d'une humanité parfaite, d'une humanité totalement rationnelle. Car Husserl, contrairement à Hegel, ne tente pas de démontrer qu'il y a de la raison dans l'histoire, mais affirme au contraire que la raison et la

\footnotetext{
${ }^{18}$ E. Husserl, La crise des sciences européennes et la phénoménologie transcendantale, trad. G. Granel, Paris, Gallimard, 1976, p. 380.

${ }^{19}$ E. Husserl, Expérience et jugement, trad. D. Souche-Dagues, Paris, PUF, 1970, § 65, p. 324 ,

${ }^{20}$ E. Husserl, La crise des sciences européennes, op. cit., p. 370.

${ }^{21}$ Passage cité par R. Toulemont in L'essence de la société selon Husserl, Paris, PUF, 1962, p. 278.
} 
vérité requièrent en elles-mêmes l'histoire. Cette nouvelle perspective transforme en fait si radicalement les notions d'histoire, de raison et de vérité qu'il devient parfois difficile de distinguer cet extrême transcendantalisme d'un simple relativisme subjectif.

Il semble donc qu'une certaine ambiguïté demeure, en ce qui concerne la question anthropologique, dans la pensée de Husserl, ce qui tendrait à confirmer la lecture qu'en fait Derrida dans «Les fins de l'homme ». On trouve en effet d'un côté l'affirmation d'une prééminence de la phénoménologie transcendantale sur toute anthropologie possible, du fait de l'absolue distinction entre la subjectivité transcendantale en tant que conscience constituante et la subjectivité psychologique en tant qu'objet constitué subsistant dans le monde ; et d'un autre côté, ce que Derrida nomme avec pertinence un « humanisme transcendantal » que Husserl développe dans sa dernière œuvre et qui implique l'idée d'une humanité produisant transcendantalement les vérités rationnelles. On peut alors comprendre que Heidegger, qui n'a pas accepté la différence établie par Husserl entre ego empirique et ego transcendantal, ait pu voir dans la théorie de la subjectivité transcendantale un retour à la "tradition de la philosophie moderne » et une nouvelle forme de la métaphysique de la subjectivité22.

Mais qu'en est-il de la position de Heidegger lui-même qui répond alors à l'accusation husserlienne $d^{\prime}$ " anthropologisme transcendantal » par une contre-accusation de « subjectivisme transcendantal »? Il est pourtant clair que la question qui a déterminé l'entièreté du « chemin de pensée » de Heidegger n'est pas celle qui a commandé toute la pensée de Husserl depuis son tournant transcendantal de 1907, à savoir celle de l'ego transcendantal, mais celle de l'être que Heidegger se propose en 1927 de reposer à neuf. Loin donc, comme Husserl le fait dans la quatrième des Méditations cartésiennes, d'identifier égologie et ontologie, en déclarant que «tout sens et tout être concevable, qu'on les dise immanents ou transcendants, relèvent du domaine de la subjectivité transcendantale en tant que c'est elle qui constitue le sens et l'être $»^{23}$, Heidegger se propose au contraire de renouer avec une question ancienne, laissée en suspens au commen-

\footnotetext{
${ }^{22}$ M. Heidegger, « Mon chemin de pensée et la phénoménologie » (1969), trad. J. Lauxerois et C. Roëls, Questions IV, Paris, Gallimard, 1976, p. 166.

${ }^{23}$ E. Husserl, Méditations cartésiennes, Paris, PUF, 1994, § 41, p. 132.
} 
cement même de la tradition occidentale, et qui, loin de ne concerner que l'être de l'homme, est au contraire celle de l'être en tant que tel et dans son ensemble. C'est d'ailleurs ce qu'il ne cessera d'opposer à ceux qui voudront l'intégrer à ce que l'on nomme Existenzphilosophie, philosophie de l'existence, de Kierkegaard à Jaspers et Sartre ${ }^{24}$. Il est vrai que le malentendu concernant le véritable statut de l'analyse existentiale dans Etre et temps qui a conduit ses premiers lecteurs à y voir la tentative de l'élaboration, d'ailleurs incomplète, d'une anthropologie philosophique a été grandement facilité par le fait que la troisième section, proprement " ontologique », de la première partie de cet ouvrage ne fut jamais publiée.

Il n'en demeure cependant pas moins que c'est encore une problématique transcendantale que Heidegger développe dans toute cette période, qui est celle de la première phase de sa pensée. Car si dans Sein und Zeit, nous ne trouvons encore qu'une analyse de l'Umwelt humain, qui répond d'ailleurs à la problématique husserlienne de la Lebenswelt, mais avec cette différence que Heidegger, contrairement à Husserl, met l'accent non sur la perception de la chose isolée, mais sur la préoccupation qui s'attache à un ensemble d'outils " maniables », c'est seulement dans ses écrits postérieurs qu'il abordera le problème de la transcendance du monde lui-même, comme il le reconnaît en 1929 dans une note de De l'essence du fondement, dans laquelle il souligne que l'analyse de l'Umwelt dans Sein und Zeit, est seulement une première détermination du phénomène du monde et n'a qu'une valeur préparatoire ${ }^{25}$. Mais c'est surtout dans le cours qu'il consacre en 1929-30 aux Concepts fondamentaux de la métaphysique: Monde, finitude, solitude, dans lequel est développée la thèse de l'homme comme configurateur du monde (weltbildend), qu'est thématisé le rapport de l'homme au monde. Or cette thèse de la Weltbildung par l'homme peut au premier abord être prise pour la thèse la plus subjectiviste qui soit, au sens où il peut sembler que le monde ne soit plus alors que le produit de l'activité configuratrice de l'homme. Ce fut en effet l'opinion de Fink, qui assista à ce cours, et auquel Heidegger, apprenant sa mort en 1975, dédia par avance ce volume 29-30 de l'édition complète de ses œuvres (Gesamtausgabe) qui ne devait paraître qu'en 1989. Fink a repris en effet à son compte, et de manière originale, la tâche que Heidegger s'était assigné en 1929, à savoir celle

\footnotetext{
${ }^{24}$ Cf. M. Heidegger, « Lettre à Jean Wahl », Bulletin de la Société française de philosophie, n 5, 1937, p. 193.

${ }_{25}$ M. Heidegger, Vom Wesen des Grundes, Francfort-sur-le-Main, Klostermann, 1955, p. 36, note 55 ; trad. H. Corbin in M. Heidegger, Questions I, Paris, Gallimard, 1968, p. 130.
} 
d'une exposition du problème du monde, dans le cadre d'un cours qu'il fit à deux reprises en 1949, puis en 1966, sous le même titre de "Monde et finitude», Welt und Endlichkeit, cours qui ne fut lui aussi publié que de manière posthume en 1990. Dans ce cours, Fink, rappelant que Heidegger tente dans Vom Wesen des Grundes d'élucider la projection transcendantale du monde en montrant qu'elle trouve son origine dans la liberté finie de l'homme, déclare que "pour le problème du monde, cet essai de Heidegger constitue le sommet d'une conception subjectiviste du monde $»^{26}$.

La configuration du monde, telle que Heidegger la comprend, ne peut cependant pas être considérée comme relevant de l'acte d'un sujet, précisément parce que le monde est toujours déjà ouvert en même temps que le Dasein lui-même. Dans Être et temps, Heidegger a en effet montré que nous avons un accès premier au monde à travers nos tonalités affectives, nos Stimmungen, et non pas à travers un acte perceptif ou intuitif, de sorte que notre être-dans-le-monde ne résulte pas d'une décision de notre part, mais est toujours déjà passivement ouvert dans cette structure existentiale de l'être de l'homme que Heidegger nomme Befindlichkeit, mot qui renvoie à la double signification du verbe sich befinden qui veut dire d'une part "sentir » et d'autre part «se situer ${ }^{27}$. Nous n'avons donc pas en fait la commande volontaire de la projection par laquelle nous nous donnons à nous-mêmes un horizon afin de rendre possible notre action et les relations que nous entretenons avec les étants. C'est la raison pour laquelle nous ne pouvons pas considérer la projection du monde comme l'acte d'un sujet transcendantal, mais nous devons plutôt l'identifier à l'avènement même de l'existence humaine.

On pourrait néanmoins considérer que la conception heideggérienne du transcendantal ne constitue dans sa nouveauté rien d'autre que la culmination de la transcendantalité kantienne ou husserlienne. $C^{\prime}$ est la raison pour laquelle Fink peut déclarer que, dans son concept transcendantal de monde, Heidegger pousse jusqu' au bout le concept kantien de monde ${ }^{28}$, jusqu'au point où elle peut se renverser en une conception véritablement cosmologique dans laquelle, précise-t-il, le monde ne dépend pas du Dasein, mais où au contraire le Dasein dépend peut-être du monde ${ }^{29}$. Une telle inversion de priorité - laquelle, il faut le souligner, ne peut avoir qu'un sens ontologique et

\footnotetext{
${ }^{26}$ E. Fink, Welt und Endlichkeit, Würzburg, Königshausen \& Neumann, 1990, p. 171.

${ }^{27}$ M. Heidegger, Sein und Zeit, Tübingen, Niemeyer, 1963, § 29, p. 137.

${ }^{28}$ Ibid., p. 154.

${ }^{29}$ Ibid., p. 183.
} 
non pas ontique - ne peut avoir lieu que si la transcendantalité n'est plus considérée comme une propriété du Dasein mais comme la dimension qui le rend possible. Or c'est précisément ce que la lecture heideggérienne de Kant tentait de faire apparaître en 1929 dans Kant et le problème de la métaphysique et en 1930 dans son cours sur De l'essence de la liberté humaine. Autrement dit, surmonter le transcendantalisme sans retomber dans l'empirisme, cela n'est rendu possible que si le transcendantal est d'ores et déjà compris en termes de facticité et d'existence, et non plus en termes d'activité et de subjectivité.

Ce qui est en effet apparu au cours de l'interprétation que Heidegger donne ici de la Critique de la raison pure, c'est que c'est dans la transcendance du sujet que se fonde la possibilité de la métaphysique, puisque, pour qu'il y ait pour lui un objet, le sujet doit le laisser surgir devant lui de par sa faculté réceptrice. Comme le souligne alors Heidegger : "L'instauration kantienne du fondement fait découvrir que fonder la métaphysique est une interrogation sur l'homme, est anthropologie ${ }^{30}$. Ce lien établi entre métaphysique et anthropologie n'est pas seulement le résultat de l'interprétation heideggérienne de la Critique de la raison pure, mais il est aussi attesté par Kant lui-même, non certes dans la Critique de la raison pure, mais dans son cours sur la Logique où, aux trois questions de la métaphysique : Que puis-je savoir? Que dois-je faire? Que m'est-il permis d'espérer?, il en ajoute une quatrième : Qu'est-ce que l'homme? Les trois premières questions forment l'objet de la metaphysica specialis, distincte de la metaphysica generalis, donc de l'ontologie, et renvoient aux trois disciplines de la cosmologie, de la psychologie et de la théologie rationnelles. Il est donc étonnant que Kant leur adjoigne une quatrième question, dont l'objet semble d'ailleurs déjà contenu dans la psychologie rationnelle, discipline qui a l'homme pour objet. Et il est encore plus étonnant que Kant précise que «les trois questions se rapportent à la quatrième » et qu'ainsi l'ensemble de la métaphysique puisse être mise «au compte de l'anthropologie »31. Car on trouve bien une anthropologie chez Kant, l'Anthropologie du point de vue pragmatique, qui rassemble la matière des cours qu'il a faits pendant de nombreuses années sur ce sujet. Mais elle ne peut ici être prise en considération, explique Heidegger, du fait qu'elle est une anthropologie empirique qui se borne à rassembler et à décrire des faits. Elle ne peut donc être considérée comme une anthropologie répondant

\footnotetext{
${ }^{30} \mathrm{M}$. Heidegger, Kant et le problème de la métaphysique, trad. A. de Waehlens et W. Biemel, Paris, Gallimard, 1953, p. 262.

${ }^{31}$ Ibid., p. 264.
} 
aux exigences de la problématique transcendantale, c'est-à-dire être un discours portant sur l'être même de l'homme.

$C^{\prime}$ est à partir de là que Heidegger s'engage dans la discussion de ce que pourrait et devrait être une anthropologie " philosophique ». Car le projet anthropologique en tant que tel suppose la prise en compte des diverses dimensions de l'humain, biologique, psychologique et culturelle, si bien que l'hétérogénéité des faits à rassembler rend impossible la définition rigoureuse d'une telle science. Le terme $\mathrm{d}^{\prime}$ « anthropologie » ne renvoie donc qu'à une étiquette qu'on appose sur un ensemble hétéroclite de disciplines, de sorte qu'il finit plutôt par désigner un point de vue explicatif qui peut être pris sur toutes choses dans la mesure où elles peuvent être mises en rapport avec l'homme. Or c'est précisément l'insatisfaction née d'une telle situation qui conduit à l'exigence d'une " anthropologie philosophique ». Heidegger se réfère ici au travail de Max Scheler, à la mémoire d'ailleurs duquel Kant et le problème de la métaphysique est dédié - Max Scheler est mort en 1928 - et il mentionne son dernier livre, paru en cette même année 1928, Die Stellung des Menschen im Kosmos, La situation de l'homme dans le cosmos, dans lequel Scheler fait état à la fois de la nécessité de parvenir à une idée unitaire de l'homme et des difficultés liées à cette tâche.

Il est nécessaire ici d'ouvrir une parenthèse, car ce projet d'élaboration d'une " anthropologie philosophique » a tout particulièrement marqué les années vingt en Allemagne, non seulement avec la parution du livre de Scheler, qui, se fondant sur l'opposition de la nature et de l'esprit, rattache l'homme à l'ordre extramondain de l'esprit, et développe une métaphysique de la personne, mais aussi avec celui que Plessner a consacré la même année sous le titre «Les niveaux du règne organique et l'homme » à une "Introduction à l'anthropologie philosophique ». Contrairement à Arnold Gehlen (1904-1976), le plus illustre représentant de ce courant de l'anthropologie philosophique, qui développera par la suite, dans un livre paru en 1940 et intitulé "L'homme, sa nature et sa place dans le monde», une conception de l'homme comme être de culture, essentiellement déterminé par son caractère inachevé et déficient, Helmuth Plessner (1892-1955), prenant appui sur les travaux des biologistes, considère que c'est dans ce que l'homme a de pleinement naturel qu'il apparaît justement comme un être extraordinaire, ce qui explique qu'il soit le seul parmi les êtres vivants à transformer son propre environnement en environnement culturel. C'est ce qu'il nomme la "positionalité excentrique » de l'homme par opposition à l'absence de centralité des plantes, dont la forme d'organisation est ouverte et dépourvue d'intériorité, et à celle, 
fermée, mais dépourvue de réflexivité des animaux, qu'il nomme «centrique». Ce qui caractérise l'humain, c'est en effet le rapport réflexif qu'il entretient avec la vie, de sorte qu'il existe sous le double rapport du corps organique (Körper) qu'il possède et du corps vivant (Leib) qu'il est. C'est donc bien cet être hors de soi (Aussersichsein) dont il fait en particulier l'expérience dans les affects et les passions, qui distingue des autres vivants l'être humain. Dans un texte plus tardif, et qui date de 1973, Plessner explique que ce rapport dialectique, cet entrecroisement (Verschränkung) du Leib et du Körper renvoie au concept d'ambiguïté propre à Merleau-Ponty ${ }^{32}$, et il affirme que, bien qu'il ait développé en 1928 son propre concept de "positionalité excentrique" sans avoir pris alors connaissance de Sein und Zeit, il n'en est pas moins compatible avec l'ontologie heideggérienne du Dasein, au sens où " la vie recèle comme l'une de ses possibilités $l^{\prime}$ existence $»^{33}$. Heidegger, qui, du moins à ma connaissance, ne cite nulle part expressément Plessner, n'en définit pas moins curieusement lui aussi, en 1929, dans une longue note de De l'essence du fondement, destinée à montrer que le reproche qui a été adressé à Sein und Zeit de promouvoir un "point de vue anthropocentrique " est une grave erreur d'interprétation, l'être du Dasein comme « ekstatique, c'est-à-dire excentrique $»^{34}$.

Il n'en reste pas moins cependant que, pour Heidegger, la difficulté fondamentale en ce qui concerne la détermination de l'être de l'homme ne consiste pas dans l'obtention d'une unité systématique de ses déterminations, mais réside dans le concept même $\mathrm{d}^{\prime}$ « anthropologie philosophique », qui demeure foncièrement ambigu. Car ce terme d'anthropologie philosophique peut signifier la recherche de ce qui constitue de manière spécifique l'être de l'homme par contraste avec celle des autres types d'étants, mais elle n'est alors qu'une ontologie régionale parmi d'autres et ne peut donc à ce titre prétendre constituer le centre de la philosophie. Il peut aussi signifier une recherche qui se fonde sur la reconnaissance de l'homme comme étant absolument premier et certain, selon la définition cartésienne, ce qui implique alors la centration de la philosophie sur la subjectivité humaine. D'une part donc l'idée d'une anthropologie philosophique s'impose irrésistiblement, mais d'autre part elle prête le flanc à des critiques qui s'attaquent à «l'anthropologisme » régnant en philoso-

\footnotetext{
${ }^{32}$ H. Plessner, «Der Aussagewert einer philosophischen Anthropologie », Mit anderen Augen, Stuttgart, Reclam, 1982, p. 142.

${ }^{33}$ Ibid., p. 134.

${ }^{34}$ M. Heidegger, Questions I, op. cit., p. 140.
} 
phie. Or toutes ces difficultés proviennent de ce que le projet «anthropologique » n'a pas été suffisamment justifié à partir de l'essence même de la philosophie et qu'il a conduit à faire de l'anthropologie, comme le souligne Heidegger, "une sorte de dépotoir de tous les problèmes philosophiques essentiels $»^{35}$.

Est-ce à dire que cette critique de l'anthropologisme que partagent, nonobstant leurs divergences de vue à cet égard, Husserl et Heidegger, signe l'impossibilité de toute anthropologie phénoménologique? Pour ce qui est de Husserl, qui a eu le projet de rendre phénoménologique cette science positive qu'est la psychologie ${ }^{36}$ - et qui a mis l'accent sur le parallélisme entre l'homme et l'ego, la psychologie intentionnelle et la phénoménologie transcendantale, il est amené, alors même qu'il fustige, dans sa conférence de 1931, toute dérive anthropologique de la phénoménologie chez ses disciples, à reconnaître que " la psychologie, et si l'on veut l'anthropologie, n'est pas une science positive parmi les autres, parmi les disciplines naturelles, mais possède une affinité intime avec la philosophie, celle qui est transcendantale ${ }^{37}$. Et il conclut sa conférence en affirmant que, si la psychologie et l'anthropologie prennent la forme d'une science rationnelle, comme ce fut le cas avec Galilée pour la science de la nature, le psychologue ou anthropologue est contraint «à abandonner sa mondanéité native pour se comprendre comme philosophe transcendantale » de sorte $\mathrm{qu}^{\prime}$ " «alors la science positive du monde vire d'ellemême en science transcendantale ${ }^{38}$. Il apparaît ainsi que la distinction husserlienne entre l'ego transcendantal et l'ego empirique, loin de barrer la route à toute enquête anthropologique, ouvre au contraire la voie à une autre compréhension de l'homme que celle qui réduit son être à celui d'un objet constitué subsistant dans le monde. Comme le souligne Philippe Cabestan, qui a commencé par interroger le sens et les limites de la psychologie phénoménologique husserlienne dans son étude sur L'être et la conscience, " n'est-ce pas en approfondissant le sens de l'intentionnalité et en renonçant à toute forme de

\footnotetext{
${ }^{35}$ M. Heidegger, Kant et le problème de la métaphysique, op. cit., p. 269.

${ }^{36}$ Voir le cours que Husserl a consacré en 1925 à la Phänomenologische Psychologie, qui a paru, accompagné de textes complémentaires, dans les Husserliana IX en 1968. Psychologie phénoménologique (1925-1928), trad. Ph. Cabestan, N. Depraz, A. Mazzu, revue par F. Dastur, Paris, Vrin, 2001.

${ }^{37}$ E. Husserl, Notes sur Heidegger, op. cit., p. 73.

${ }^{38}$ Ibid., p. 73-74.
} 
naturalisme que la psychologie phénoménologique (et donc l'anthropologie) pourrait prendre un nouveau départ? $»^{39}$ Or c'est précisément à cet approfondissement de l'intentionnalité que procédait Heidegger dans De l'essence du fondement, un essai dédié à Husserl pour son soixante-dixième anniversaire et son départ à la retraite, où il affirmait que l'intentionnalité, c'est-à-dire le rapport à l'étant particulier, n'est possible que sur le fondement de la transcendance du Dasein vers le monde, et non le contraire ${ }^{40}$. Ce qui en résulte, $c^{\prime}$ est une différence encore plus tranchée entre la constitution de ce qui n'est plus un sujet, mais un Dasein et un être "configurateur » du monde, et la constitution de la nature, de sorte que, affirme Heidegger dans son cours de 1927 sur Les problèmes fondamentaux de la phénoménologie, "existence et être-subsistant (Vorhandenheit) sont plus disparates que ne le sont, par exemple, dans l'ontologie traditionnelle, les déterminations de l'être de Dieu et celle de l'homme ${ }^{41}$.

C'est cette «différence ontologique » que Heidegger va creuser toujours davantage au cours d'un chemin de pensée qui l'éloignera de plus en plus d'une pensée de l'existence de l'homme représentée à partir de la subjectivité et de la substance ${ }^{42}$. Il engage ainsi la pensée sur la voie d'une toute autre expérience de l'être de l'homme que celle qui commande aujourd'hui les représentations que l'on se fait de l'homme dans ces disciplines aujourd'hui devenues dominantes que l'on nomme «sciences cognitives » ou «neurosciences». Car la rupture radicale qu'il opère avec l'anthropocentrisme qui régit depuis son début la pensée moderne ne signifie nullement l'abandon pur et simple de la question de l'homme. C'est en substance ce que Heidegger expliquait au cours d'une émission de télévision organisée en septembre 1969 à l'occasion de son quatre-vingtième anniversaire. Au professeur Richard Wisser qui lui demandait si sa philosophie pouvait avoir une action concrète sur la société ou si au contraire il fallait donner raison à ceux de ses critiques qui prétendent qu'il a sacrifié au souci de l'être la condition humaine et l'être de l'homme en société et en tant que personne, Heidegger répondait que cette critique provenait d'un grand malentendu sur sa pensée, du fait «qu'on ne peut pas poser la question de l'être sans poser celle de l'homme ${ }^{43}$.

\footnotetext{
${ }^{39}$ Ph. Cabestan, L'être et la conscience, Bruxelles, Ousia, 2004, p. 32.

${ }^{40}$ M. Heidegger, Questions I, op. cit., p. 101.

${ }^{41}$ M. Heidegger, Les problèmes fondamentaux de la phénoménologie, Paris, Gallimard, 1985, §15, p. 216.

${ }_{42}$ M. Heidegger, Questions I, op. cit., p. 34.

43 « Entretien du Professeur Richard Wisser avec Martin Heidegger (1969) », in Martin Heidegger, Paris, L'Herne, 1983, p. 94.
} 
Pas plus en effet qu'on ne peut trouver chez le dernier Heidegger l'idée que l'être aurait la capacité de se manifester par lui-même en employant l'homme comme un simple medium, on ne trouve chez le premier Heidegger l'idée contraire selon laquelle l'être ne serait que le résultat du projet d'un Dasein conçu sur le modèle d'un sujet transcendantal. Ce qu'il a cherché, sans doute dès le début, à développer, c'est, sous le nom de ce qu'il nommera, à partir du milieu des années trente, "Ereignis », une nouvelle conception de l'être, non plus considéré comme le fondement des étants, mais comme l'événement, à partir d'une occultation abyssale, d'une éclaircie qui n'est pas le fait de l'homme, mais dans laquelle celui-ci se tient. Dans la perspective de cette nouvelle conception, l'homme n'est plus considéré comme le fondement jeté de l'éclaircie, mais comme celui qui demeure exposé à elle et qui lui est redevable de son propre être. L'être en tant que tel et l'être de l'homme ne coïncident plus. Le «là » de l'être ne peut plus être compris comme le résultat de la projection et de la transcendance de l'être de l'homme, mais comme l'adresse (Anspruch) de l'être à l'homme, à laquelle l'homme a à répondre et à co-respondre (entsprechen). Le Verhältnis, le rapport de l'être à l'homme, ne doit pas être compris comme une simple relation entre deux entités séparées, mais comme la manière selon laquelle l'être se retire lui-même en laissant être le Da-sein : au retrait de l'être, l'homme non métaphysique répond par sa réserve, sa Verhaltenheit, laquelle devient la tonalité fondamentale (Grundstimmung) de son être. Heidegger donne le nom d'Ereignis à cette co-appartenance de l'être et de l'homme, qui n'est ni coïncidence, ni relation dialectique, mais être l'un pour l'autre et constellation de l'homme et de l'être ${ }^{44}$.

Mais si pour Heidegger, la question de l'être de l'homme n'a rien à voir avec cette science positive qui se nomme " anthropologie », mais fait au contraire inséparablement partie de la question de l'être, quel pourrait donc bien être le contenu d'une « anthropologie phénoménologique », c'est-à-dire d'un discours sur l'homme qui ne verrait en lui qu'un être parmi d'autres, mais celui qui prend part à « l'événement » de l'éclaircie, lequel constitue le phénomène fondamental de la «phénoménologie de l'inapparent » heideggérienne ${ }^{45}$ ? La réponse pourrait être la même que celle que Heidegger a donnée à la question posée en 1946 par Jean Beaufret concernant la relation de l'ontologie à une éthique possible. Heidegger avait commencé par montrer que

\footnotetext{
${ }^{44}$ M. Heidegger, «Identité et différence » (1957), Questions I, op. cit., p. 267 et 270.

${ }^{45}$ Cf. M. Heidegger, « Le séminaire de Zähringen » (1973), Questions IV, Paris, Gallimard, 1976, p. 339.
} 
penser en termes de disciplines séparées n'est pas approprié, d'abord parce que sa propre pensée ne peut pas être désignée par le terme d'ontologie, car c'est là le nom d'une discipline traditionnelle dont le sujet est l'étantité de l'étant et non la vérité de l'être, à savoir l'aletheia, l'avènement de l'éclaircie. Il en va de même concernant l'éthique, qui a également été définie, avec la logique et la physique, comme les disciplines formant le concept traditionnel de philosophie, de sorte que la sphère de l'action humaine a été considérée comme un domaine séparé de la connaissance. Heidegger, rappelant que le mot ethos signifie originellement «lieu d'habitation » en grec, explique que la «pensée qui pense la vérité de l'être comme l'élément originel de l'homme en tant qu'eksistant est déjà en elle-même l'éthique originelle $»^{46}$.

Une fois que l'homme n'est plus placé au centre de l'étant, comme ce fut le cas dans la philosophie moderne depuis Descartes, il devient alors impossible de considérer que le sujet principal de la pensée est constitué par l'éthique, qui ne concerne que les relations des êtres humains entre eux, ou par l'anthropologie, qui se limite à la sphère humaine comme telle. Déjà dans les années trente, Heidegger déclarait que «la philosophie qui a cours à l'époque de la métaphysique achevée est l'anthropologie ${ }^{47}$. Il semble que ce soit plus que jamais le cas aujourd'hui, puisque la réaction anti-humaniste de la philosophie des années soixante $\mathrm{n}^{\prime}$ a pas duré très longtemps, comme le montre bien le fait que Foucault et Derrida ont fait retour, à la fin de leur itinéraire philosophique respectif, à des problèmes éthiques, politiques et anthropologiques, la question demeurant à cet égard de savoir s'ils en ont véritablement traité autrement que sur des bases purement empiriques ${ }^{48}$. Heidegger, au début du $X X^{\mathrm{e}}$ siècle, a, quant à lui, renoué avec la toute première orientation de la philosophie, laquelle avait trouvé son expression dans la sentence d'un des plus anciens penseurs occidentaux, Périandre de Corinthe, un des sept sages de la Grèce, dont, dans ses cours sur Nietzsche de la fin des années trente, Heidegger rappelait l'injonction : Meleta to pan, «Soucie-toi de l'étant dans sa totalité ${ }^{49}$. Il nous incitait ainsi à faire retour,

\footnotetext{
${ }^{46}$ M. Heidegger, Lettre sur l'humanisme, Paris, Aubier Montaigne, 1964, p.151.

${ }^{47}$ M. Heidegger, «Dépassement de la métaphysique », Essais et conférences, Paris, Gallimard, 1958, p. 99.

${ }^{48}$ Voir en particulier les textes suivants, dont les titres sont particulièrement explicites : J. Derrida, Force de loi (1990), Politiques de l'amitié (1994), Donner la mort (1995), De l'hospitalité (1997) ; M. Foucault, Surveiller et punir (1975), Histoire de la sexualité (1976-1984), L'herméneutique du sujet (2001).

${ }^{49}$ Cf. M. Heidegger, Nietzsche I, trad. P. Klossowski, Paris, Gallimard, 1971, p. 371.
} 
contre le positivisme toujours dominant, à ce qui constitue la spécificité de l'homme, à savoir la relation à ce qui n'est pas humain, laquelle fait partie de manière aussi fondamentale de l'être humain que sa relation aux autres êtres humains. Au commencement de ce XXI ${ }^{e}$ siècle, et devant les défis auxquels l'humanité se voit aujourd'hui confrontée sans pouvoir désormais ne se préoccuper exclusivement que d'elle-même - car il lui faut désormais prendre en charge la destinée de la planète toute entière -, cette ancienne injonction devrait trouver en nous un écho toujours plus grand. 